\title{
Validation of a predictive rule for the management of community-acquired pneumonia
}

\author{
A. Capelastegui*, P.P. España*, J.M. Quintana\#, I. Areitio", I. Gorordo*, \\ M. Egurrola* and A. Bilbao ${ }^{+}$
}

ABSTRACT: The CURB-65 score (Confusion, Urea $>7 \mathrm{mmol} \cdot \mathrm{L}^{-1}$, Respiratory rate $\geqslant 30 \cdot \mathrm{min}^{-1}$, low Blood pressure, and age $\geqslant 65 \mathrm{yrs}$ ) has been proposed as a tool for augmenting clinical judgement for stratifying patients with community-acquired pneumonia (CAP) into different management groups.

The six-point CURB-65 score was retrospectively applied in a prospective, consecutive cohort of adult patients with a diagnosis of CAP seen in the emergency department of a 400-bed teaching hospital from March 1, 2000 to February 29, 2004. A total of 1,100 inpatients and 676 outpatients were included.

The 30-day mortality rate in the entire cohort increased directly with increasing CURB-65 score: 0,1.1, 7.6, 21, 41.9 and $60 \%$ for CURB-65 scores of $0,1,2,3,4$, and 5 , respectively. The score was also significantly associated with the need for mechanical ventilation and rate of hospital admission in the entire cohort, and with duration of hospital stay among inpatients.

The CURB-65 score (Confusion, Urea $>7 \mathrm{mmol} \cdot \mathrm{L}^{-1}$, Respiratory rate $\geqslant 30 \cdot \mathrm{min}^{-1}$, low Blood pressure, and age $\geqslant 65 \mathrm{yrs}$ ), and a simpler CRB-65 score that omits the blood urea measurement, helps classify patients with community-acquired pneumonia into different groups according to the mortality risk and significantly correlates with community-acquired pneumonia management key points. The new score can also be used as a severity adjustment measure.

KEYWORDS: Community-acquired pneumonia, prediction rule, severity assessment

A key step in the management of community-acquired pneumonia (CAP) is the initial assessment of the severity of the disease. An accurate assessment helps the clinician determine the site of care, the extent of diagnostic testing, and the type and intensity of antibiotic treatment. However, a number of studies suggest that routine clinical judgement is often not sufficient for assessing the severity of CAP. Clinical judgement alone may underestimate its severity [1] and lead to variations in rates of admission to the hospital [2,3] and intensive care unit (ICU) [4]. In addition, the decision to admit a patient to the ICU based on clinical judgement alone has been found to be suboptimal [5]. In this light, validated clinical prediction rules for CAP management offer a useful adjunct to the art of clinical practice.

The Pneumonia Severity Index (PSI) [6], developed in the USA, has been shown to be a

For editorial comments see pages 6 and 9. powerful tool for predicting pneumonia mortality in different countries [6-8]. It can be used to predict the probability of death and other relevant medical outcomes within different risk classes. Because determining the PSI requires 20 criteria, it is advisable to use specific software for its routine application in hospital emergency departments (ED). The determination of the PSI in the primary care setting is more problematic, given that the analytic determinations necessary for its calculation are not always available.

An international study conducted in Europe [9] proposed a new clinical prediction rule, the CURB-65 score (Confusion, Urea $>7 \mathrm{mmol} \cdot \mathrm{L}^{-1}$, Respiratory rate $\geqslant 30 \cdot \mathrm{min}^{-1}$, low Blood pressure, and age $\geqslant 65 \mathrm{yrs}$ ). It uses a six-point scale, ranging from 0 to 5 . This score, based on a modification of an earlier CURB score [1, 10], facilitated the separation of patients into three management groups with mortalities ranging from 0 to $33 \%$ in the derivation cohort [9]. The basic information required to determine the
AFFILIATIONS

* Service of Pneumology,

${ }^{*}$ Research Unit, and

"Dept of Emergency Medicine

Hospital de Galdakao, Galdakao, and

${ }^{+}$The Basque Foundation for Health Innovation and Research (BIOEF),

Sondika, Bizkaia, Spain.

CORRESPONDENCE

A. Capelastegui

Service of Pneumology

Hospital de Galdakao

E-48960 Galdakao

Bizkaia

Spain

Fax: 34944572327

E-mail:

acapelas@hgda.osakidetza.net

Received:

May 302005

Accepted after revision:

October 162005
European Respiratory Journal Print ISSN 0903-1936 Online ISSN 1399-3003 
CURB-65, which is available at the initial hospital assessment, could provide objective support for clinical decision-making regarding the need for hospital admission or intensive care management. A similar tool that omits the blood urea measurement (the CRB-65 score) could be used in the community.

The aim of the current study was to evaluate the ability of the CURB-65 score to stratify patients with CAP into different management groups. A large prospective cohort was employed that included all patients diagnosed with CAP in the ED of a general teaching hospital over a 4-yr period.

\section{METHODS}

\section{Setting of study and design}

The current study was carried out in Galdakao Hospital (Galdakao, Spain), a 400-bed teaching hospital in the Basque Country (northern Spain) that serves a population of 300,000 inhabitants. This medical institution belongs to the network of public hospitals of the Basque Health Care Service, which provides free unrestricted care to nearly $100 \%$ of the population. The hospital staff is composed of full time physicians of various specialities qualified in standardised national residency programs. The hospital's ICU is an independent service assisted by specialised physicians. The ED staff is composed of full-time physicians of diverse specialities, including ED specialists and primary care physicians. During the study, a guideline for the management of patients with CAP (described in more detail elsewhere [7]) was implemented. Explicit admission-decision criteria were established based on risk classes defined according to the PSI [6] with a series of additional criteria [7].

\section{Study population}

The study population was composed of a consecutive cohort of adults aged $\geqslant 18$ yrs admitted to the hospital's ED with a diagnosis of CAP from March 1, 2000 to February 29, 2004. Only individuals in whom CAP was suspected within the first $24 \mathrm{~h}$ after arrival at the ED were included. Pneumonia was defined as pulmonary infiltrates on chest radiograph not known to be old and symptoms that were consistent with pneumonia, including cough, dyspnoea, fever, and/or pleuritic chest pain. Patients with pneumonia were excluded if they were known to be positive for HIV, were chronically immunosuppressed (defined as immunosuppression for solid organ transplantation, post-splenectomy, receiving $\geqslant 10 \mathrm{mg} \cdot \mathrm{day}^{-1}$ of prednisone or the equivalent for $>30$ days, treatment with other immunosuppressive agents, or neutropenic, i.e. $<1.0 \times 10^{9} \cdot \mathrm{L}^{-1}$ neutrophils), or had been hospitalised for the previous 14 days.

\section{Patient characteristics and assessment of indicators}

Clinical and demographic characteristics of each patient were recorded, along with previous antibiotic treatment and variables needed to determine both the PSI and CURB-65 scores. To measure the severity of CAP, the PSI, CURB-65 and CRB-65 scores were independently calculated. For calculating the PSI score, all missing data or unperformed laboratory tests were considered to be normal. Classes of PSI, CURB-65 and CRB-65 were created according to the original authors' classifications $[6,9]$. All patients were treated with empirical antibiotics according to local guidelines: $\beta$-lactam with macrolide, levofloxacin, or $\beta$-lactam monotherapy.

For patients initially treated as outpatients, the ED staff provided a telephone number to contact a member of the research team and a list of primary care providers. A follow-up telephone call was made within 3-5 days of the ED visit. Subsequent hospital admissions due to pneumonia-related complications and vital status within 4 weeks were recorded. Outpatients hospitalised within 4 weeks of the initial ED visit were included in the inpatient cohort. For inpatients, the following indicators were recorded: total duration of antibiotic therapy, duration of intravenous antibiotic therapy, vital status after discharge and within 4 weeks, admission to the ICU and use of a mechanical ventilator, hospital readmission within 4 weeks due to pneumonia-related complications, and length of stay (calculated as the discharge date minus the admission date, in-hospital deaths were excluded). Vital status was evaluated by use of medical records and a regional administrative database. Medical records of readmitted patients were evaluated independently by two trained pneumologists.

The project was approved by the hospital's ethics review board.

\section{Statistical analysis}

Descriptive statistics of sociodemographic and clinical variables included frequencies, percentages, means and standard deviations (SD). Comparisons of differences in variables between groups with different CURB-65 and CRB-65 scores were performed using the Chi-squared test or Fisher's exact probability test for categorical variables. Either ANOVA, using Scheffe's method for multiple comparisons, or the KruskalWallis test were used for continuous variables. Receiveroperating characteristic curves were calculated for the PSI, CURB-65, and CRB-65 scores to evaluate how well the scores discriminated between patients who survived CAP and those who died [11]. The area under the curve (AUC) and 95\% confidence intervals are given. Values were compared by using the nonparametric method described by HANLEY and MCNEIL [12]. A two-tailed p-value $<0.05$ was considered statistically significant.

\section{RESULTS}

A total of 1,776 patients, 1,100 inpatients (61.9\%) and 676 outpatients $(38.1 \%)$, were included in the study. Of these, 1,724 $(97.1 \%)$ had datasets for all risk scores under evaluation (data were missing for $>1 \%$ of patients for all variables). The mean (SD) age was 61.8 yrs (20.5; range 18-96); a total of $973(54.8 \%)$ were aged $\geqslant 65$ yrs. Baseline characteristics of the patients and PSI risk classes are shown in table 1 . The rates of mortality among inpatients and outpatients were $0 \%$ for PSI risk class I, $0.4 \%$ for risk class II, $2.1 \%$ for risk class III, $8.5 \%$ for risk class IV and $38.3 \%$ for risk class $\mathrm{V}$. Of the 1,100 patients admitted to the hospital, 484 (44\%) were assigned to low-risk PSI classes (I-III). Inpatient care was justified in $313(64.7 \%)$ of these patients because criteria for admission decision were present. In the remaining 171 (35.3\%) patients, although specific criteria for hospitalisation were absent, they were hospitalised according to the personal judgment of the emergency physicians. 


\section{TABLE 1 Characteristics of patients}

\begin{tabular}{lccc} 
Characteristics & Inpatients & Outpatients & All patients \\
\hline Subjects n & 1100 & 676 & 1776 \\
Male & $715(65)$ & $409(60.5)$ & $1124(63.3)$ \\
Age yrs & $71.1 \pm 15.9$ & $46.8 \pm 17.9$ & $61.8 \pm 20.5$ \\
Age $\geqslant$ 65 yrs & $823(74.8)$ & $150(22.2)$ & $973(54.8)$ \\
Nursing home resident & $99(9.0)$ & $3(0.4)$ & $102(5.7)$ \\
Prior antibiotics & $210(19.8)$ & $126(19.8)$ & $336(19.8)$ \\
Co-morbid illnesses & & & \\
$\quad$ Neoplastic disease & $70(6.4)$ & $2(0.3)$ & $72(4.1)$ \\
Liver disease & $55(5.0)$ & $7(1.0)$ & $62(3.5)$ \\
Congestive heart failure & $90(8.2)$ & $11(1.6)$ & $101(5.7)$ \\
Cerebrovascular disease & $135(12.3)$ & $9(1.3)$ & $144(8.1)$ \\
$\quad$ Renal disease & $106(9.6)$ & $9(1.3)$ & $115(6.5)$ \\
PSI risk class ${ }^{\#}$ & & & \\
I & $113(10.3)$ & $407(60.2)$ & $520(29.3)$ \\
II & $111(10.1)$ & $176(26)$ & $287(16.2)$ \\
III & $260(23.6)$ & $78(11.5)$ & $338(19)$ \\
IV & $423(38.5)$ & $15(2.2)$ & $438(24.7)$ \\
$\quad$ V & $193(17.6)$ & $0(0)$ & $193(10.9)$ \\
PSI & $98.8 \pm 35.5$ & $46.6 \pm 21.2$ & $78.9 \pm 39.9$ \\
\hline
\end{tabular}

Data presented as $n(\%)$ or mean \pm SD. ${ }^{*}$ : severity of illness was assessed using the Pneumonia Severity Index (PSI) [6]. Class I patients have the lowest severity and mortality risk, and class $\mathrm{V}$ the highest severity and mortality risk.

The 30-day mortality rate in the entire cohort was $6.7 \%$ (119/ $1,776)$; among inpatients it was $10.7 \%(118 / 1,100)$. In the inpatient group, 46 (40\%) died from pneumonia as an expected terminal event of a chronic disabling illness. A total of 45 patients were admitted to the ICU, 18 (40\%) of whom required mechanical ventilation. Among those admitted to the ICU, the 30 -day mortality rate was $11.1 \%(5 / 45)$. The mean age of the patients admitted to the ICU was 60.6 yrs. Among patients aged $\geqslant 65 \mathrm{yrs}, 2.9 \%$ were admitted to the ICU.

The predictive potential of the CURB-65 score for 30-day mortality, mechanical ventilation and hospital admission are shown in table 2. Mortality increased directly with the CURB65 score $(\mathrm{p}<0.001)$. The mortality rate for a CURB-65 score of 3-5 was $>20 \%$. The rate of patients admitted to the hospital also increased directly with the CURB-65 score $(\mathrm{p}<0.001)$.

The ability of the CRB-65 to predict 30-day mortality, mechanical ventilation and hospital admission was also evaluated. As with CURB-65 scores, CRB-65 was also significantly correlated with 30-day mortality, mechanical ventilation, and hospital admission (see table 2).

The receiver-operating characteristic curves of mortality prediction are shown in figure 1. The AUC of the CURB-65 was similar to the CRB-65 (AUC 0.870 versus $0.864, \mathrm{p}=0.44$ ) and to the PSI (AUC 0.870 versus $0.888, p=0.12$ ) with regard to prediction of pneumonia mortality. The results were similar when the 46 patients who died from pneumonia as an expected terminal event of a chronic disabling illness were excluded.

Table 3 shows the influence of additional criteria for hospital admission in patients with CURB-65 scores of 0,1 and 2. In both in- and outpatient groups, the presence of coexisting disease, hypoxaemia, bilateral or multilobe radiographic involvement, and pleural effusion increased directly with the CURB-65 score $(p<0.05)$. The proportion of patients with one or more additional criteria for hospital admission decision was $22.7 \%$ for a CURB-65 score of $0,53.6 \%$ for a score of 1 , and Respiratory rate, Blood pressure, and age $\geqslant 65 \mathrm{yrs}$ ) score

\begin{tabular}{|c|c|c|c|c|}
\hline & Patients n & 30-day mortality & Mechanical ventilation ${ }^{\#}$ & Admission to hospital \\
\hline \multicolumn{5}{|c|}{ CURB-65 score } \\
\hline 1 & 377 & $4(1.1)$ & $2(0.5)$ & $247(65.5)$ \\
\hline 2 & 474 & $36(7.6)$ & $9(1.9)$ & $406(85.7)$ \\
\hline 3 & 224 & $47(21)$ & $4(2)$ & $222(99.1)$ \\
\hline Total & 1776 & $119(6.7)$ & $18(1)$ & $1100(61.9)$ \\
\hline$p$-value & & $<0.001$ & $<0.001$ & $<0.001$ \\
\hline \multicolumn{5}{|c|}{ CRB-65 score } \\
\hline 0 & 716 & $0(0)$ & $1(0.1)$ & $201(28.1)$ \\
\hline 1 & 686 & $28(4.1)$ & $8(1.2)$ & $529(77.1)$ \\
\hline$p$-value & & $<0.001$ & $<0.001$ & $<0.001$ \\
\hline
\end{tabular}

Data presented as $\mathrm{n}(\%)$ and included all patients (both inpatients and outpatients). ${ }^{*}$ : deaths from pneumonia as an expected terminal event of a chronic disabling illness excluded. 


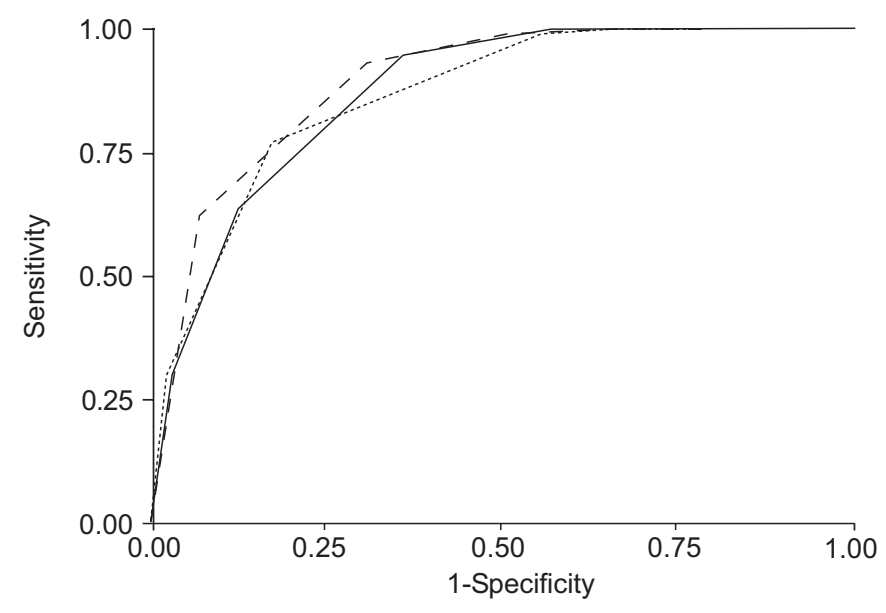

FIGURE 1. Receiver-operating characteristic curves of predicting 30-day mortality according to pneumonia severity index (PSI), CURB-65 and CRB-65 (Confusion, Urea, Respiratory rate, Blood pressure, and age $\geqslant 65 \mathrm{yrs}$; see text for exact definition) score. - PSI (area under the curve (AUC) $0.888,95 \%$ confidence interval (Cl) 0.864-0.912); - ---: CURB-65 (AUC 0.870, 95\% Cl 0.8440.895); ------: CRB-65 (AUC 0.864, 95\% Cl 0.835-0.892).

$72.4 \%$ for a score of 2 . More than $26 \%$ of inpatients with CURB65 scores of 1 and 2 had two or more additional criteria. The admitted patients have more of the additional criteria than those not admitted $(\mathrm{p}<0.01)$.

Table 4 shows the distribution of CURB-65 scores according to PSI. Among patients with CURB-65 scores of 0 and 1,92.4\% (929) were in low-risk PSI classes (I-III) with 30-day mortality rates of $0.2 \%$. The 474 patients with CURB-65 scores of 2 were distributed in two subgroups, with statistically significant $(\mathrm{p}<0.001)$ differences in 30 -day mortality: $40.9 \%$ in PSI risk classes I-III (2.6\% 30-day mortality), and $59.1 \%$ in PSI risk classes IV-V (11.1\% 30-day mortality). Among patients with CURB-65 scores of 3-5, 92.6\% (274/296) belonged to PSI risk class IV-V, with 30 -day mortality rates of $28.5 \%$.

Inpatient medical indicators by CURB-65 scores are shown in table 5. In-hospital mortality and 30-day mortality increased directly with CURB-65 scores $(\mathrm{p}<0.001)$. The results were similar when the 46 patients died from pneumonia as an expected terminal event of a chronic disabling illness were excluded. There was a significant association between the CURB-65 score and other medical indicators. The duration of intravenous antibiotic therapy ranged from a mean of 2.6 days for patients with CURB-65 scores of 0-5.6 days for patients with CURB-65 scores of $5(\mathrm{p}<0.001)$. The percentage of patients hospitalised for $<4$ days was $44.8 \%$ for those with CURB-65 scores of 0 and $1,40.1 \%$ for CURB- 65 scores of 2 , and $28.9 \%$ for CURB-65 scores $>2(\mathrm{p}<0.001)$. Mechanical ventilation was required for $0.5 \%$ of patients with CURB-65 scores of 0 and 1 , $2.3 \%$ of those with CURB-65 scores of 2, and 2.7\% for CURB-65 scores $>2(p<0.05)$. The rate of subsequent hospitalisation within 30 days was $1.3 \%$ for patients with CURB-65 scores of 0 and $1,3.5 \%$ for those with CURB-65 scores of 2 , and $4.8 \%$ for those with CURB-65 scores $>2(\mathrm{p}<0.05)$.

The proportion of patients initially treated as outpatients and subsequently hospitalised was $4.4 \%$; none of these patients died, and only one was admitted to an ICU. The 30-day mortality rate in outpatients was $0.1 \%$.

TABLE 3 Additional criteria for admission decision in patients with CURB-65 (Confusion, Urea, Respiratory rate, Blood pressure,
and age $\geqslant 65$ yrs) scores of 0,1 , and 2

\begin{tabular}{|c|c|c|c|c|c|}
\hline \multicolumn{6}{|l|}{ Outpatients and inpatients } \\
\hline Patients n & 629 & 377 & 474 & 1480 & \\
\hline Coexisting disease & 3.5 & 22.8 & 34.2 & 18.2 & $<0.001$ \\
\hline $\mathrm{Pa}, \mathrm{O}_{2}<60 \mathrm{mmHg}$ & 7.5 & 28.7 & 42.4 & 24.1 & $<0.001$ \\
\hline Bilateral or multilobe radiographic involvement & 9.2 & 14.1 & 21.1 & 14.3 & $<0.001$ \\
\hline Pleural effusion & 6 & 8.2 & 11 & 8.2 & 0.01 \\
\hline One of previous criteria & 18.1 & 35.5 & 39.5 & 29.4 & $<0.001$ \\
\hline \multicolumn{6}{|l|}{ Inpatients } \\
\hline Patients $n$ & 153 & 247 & 406 & 806 & \\
\hline Coexisting disease & 9.2 & 30 & 36.2 & 29.2 & $<0.001$ \\
\hline $\mathrm{Pa}, \mathrm{O}_{2}<60 \mathrm{mmHg}$ & 22.2 & 40.1 & 47.8 & 40.6 & $<0.001$ \\
\hline $\mathrm{pH}<7.35$ & 3.3 & 0.8 & 5.4 & 3.6 & $<0.01$ \\
\hline Bilateral or multilobe radiographic involvement & 26.1 & 19.4 & 23.9 & 23 & 0.25 \\
\hline
\end{tabular}


TABLE 4 Distribution of CURB-65 (Confusion, Urea, Respiratory rate, Blood pressure, and age $\geqslant 65$ yrs) scores according to the pneumonia severity index (PSI) and subgroup 30-day mortality

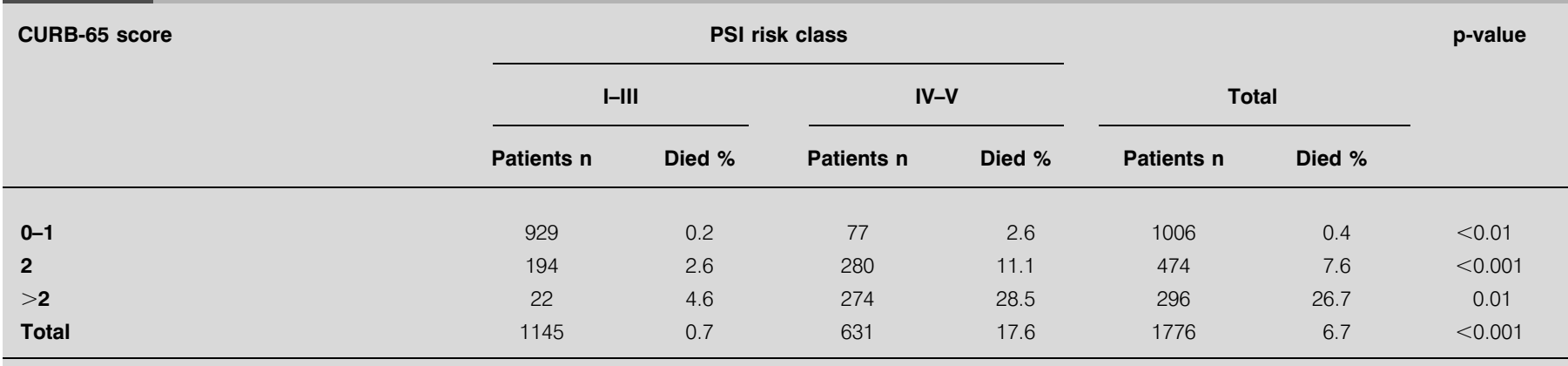

Total patients includes both inpatients and outpatients.

TABLE 5 Inpatient medical indicators by CURB-65 (Confusion, Urea, Respiratory rate, Blood pressure, and age $\geqslant 65$ yrs) scores

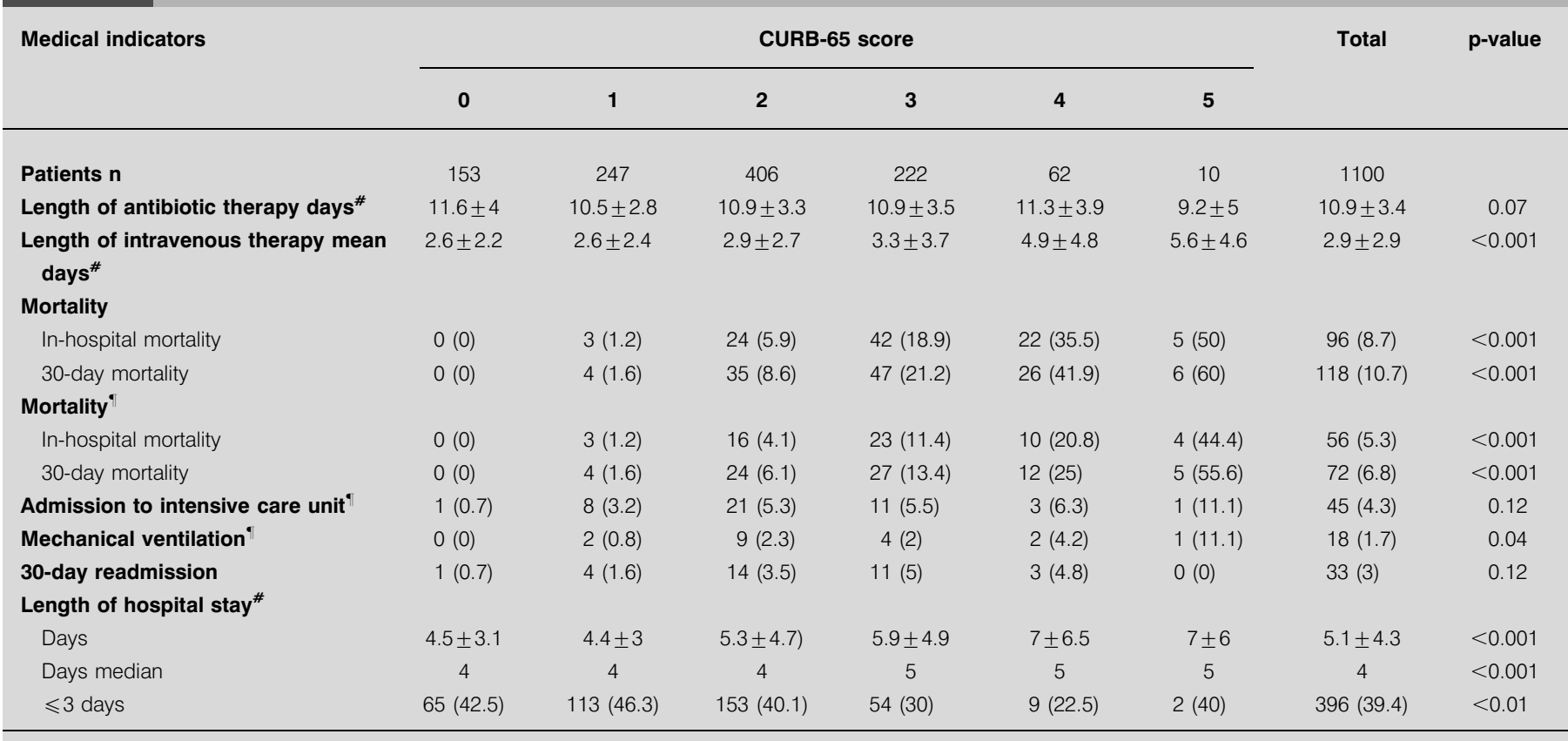

Data are presented as mean \pm SD or $\mathrm{n}(\%) .{ }^{\#}$ : in-hospital deaths excluded. ": deaths from pneumonia as an expected terminal event of a chronic disabling illness excluded.

\section{DISCUSSION}

The current study demonstrates a significant correlation between the CURB-65 score and the risk of 30-day mortality, need for mechanical ventilation, and rate of hospital admission. Similar results were obtained with the even simpler CRB65 score. Among hospitalised patients, the CURB-65 score was significantly associated with duration of hospital stay.

The strength of the current study is that it included a large cohort of both inpatients and outpatients. This made it possible to assess the utility of the CURB-65 score in assisting the decision for hospital admission. The patients were unselected adults of all ages seen in the ED of a general teaching hospital with a wide catchment area. Data for each patient was recorded prospectively, which allowed the current authors to compile relevant clinical information. Furthermore, the profile of the patient population was comparable with the one in which the CURB-65 was developed and evaluated [9].

A recent study has shown that the PSI has a higher discriminatory power for predicting 30-day mortality than the CURB-65 score [13]. It was observed that CURB-65 scores were quite similar to those from the PSI scoring system with regard to the prediction of pneumonia global mortality. Differences between the two studies may be due to the fact that the sample of AUJESKY et al. [13] may not reflect the full prognostic spectrum of patients with pneumonia because these investigators did not enrol more severely ill patients. The current study also shows that the CURB-65 score allowed for the stratification of inpatients according to increasing mortality 
risk and was correlated with the use of mechanical ventilation. However, the correlation observed between CURB-65 scores and ICU admission was not significant. This may be due to the low rate of ICU admission in this population (4.3\%), which was slightly lower than the $5 \%$ rate in the study by LIM et al. [9] and much lower than the rates of $10-18 \%$ reported in the literature [14].

Although there is no good indicator for assessing the appropriateness of the decision regarding ICU admission, some data do suggest underuse of the ICU. The percentage of patients aged $\geqslant 65 \mathrm{yrs}$ admitted to the ICU $(2.9 \%)$, the mean age of the total number of patients admitted to the ICU (60.6 yrs) and the mortality rate among patients admitted to ICU $(11.1 \%)$ are lower than those reported in other studies [15, 16]. In the current study, older age may have been used as a restrictive criterion for ICU admission. Nevertheless, accumulating evidence suggests that care given to critically ill elderly patients in the ICU is justified [17, 18]. Underuse of the ICU also can be attributed to differing medical attitudes about the treatment of patients with severity criteria who do not require mechanical ventilation and/or vasopressors, as well as to the structural organisation of the services of the author's hospital. In any case, the decision to admit a patient to the ICU is always a clinical one.

The data in the current study show that the CURB-65 score can be used to augment clinical judgement regarding the need for hospital admission. Patients with CURB-65 scores $>2$ had high mortality rates and required admission to the hospital. Most patients with CURB-65 scores of 2 need admission to the hospital; in the current study, $72.4 \%$ of patients in this category (78.5\% of those admitted to hospital) had one or more additional adverse prognosis criteria. The new CURB-65 score, in contrast with the previous CURB score [10, 19], divides mild patients into two subgroups (score 0 and 1) with a slightly different behaviour. Most patients with CURB-65 scores of 0 had excellent outcomes (no deaths and only $0.7 \%$ admitted to the ICU) and most did not require hospital care $(75.7 \%$ treated as outpatients). However, patients with CURB-65 scores of 1 need additional assessment to determine whether they should be hospitalised. In fact, $53.5 \%$ of these patients $(68.8 \%$ of those admitted to hospital) had one or more additional adverse prognosis criteria. Therefore, the findings herein emphasise the importance of assessing the presence of other adverse features, such as the presence of coexisting disease, hypoxaemia, arterial $\mathrm{pH}<7.35$, the extent of radiographic shadowing and the presence of pleural effusion, among patients with CURB-65 scores of 1 or 2 in order to identify those suitable for home treatment. This approach is in agreement with the British Thoracic Society guidelines for cases in which the CAP risk assessment is unclear [10]. In all instances, the admission decision remains an "art of medicine" decision, and $\geqslant 30 \%$ of "low-risk" patients require hospital admission [20].

The length of stay for CAP inpatients depends on the time needed to reach clinical stability, which is significantly influenced by the severity of disease [21]. Patients with the most severe pneumonia take the longest to recover and remain in the hospital for longer periods. The current study showed that CURB-65 scores, like the PSI index $[6,21]$, can be used to estimate the length of time for clinical improvement and the duration of hospital stay. The assessment of the severity of illness at the time of hospital admission, as defined by the CURB-65 score, can help physicians determine which patients may be candidates for an early switch to oral antibiotic therapy and early discharge. Over $40 \%$ of patients with CURB-65 scores of 0,1 and 2 stayed in the hospital for $\leqslant 3$ days. The mean duration of intravenous therapy and hospital stay in the current study was consistent with that in studies published elsewhere [22, 23].

One limitation of the current study is that the potential effect of applying the CURB-65 score was assessed only among a consecutive cohort of adults admitted to the hospital's ED with a diagnosis of CAP rather than assessing its use as the main admission criterion for all patients with CAP.

It has recently been shown that implementing a guideline for the management of patients hospitalised for the treatment of CAP significantly improves the process-of-care and outcomes [24]. Others have demonstrated similar improvements [25-27]. The current study identified two areas for improvement: the proportion of patients admitted to the hospital (61.9\%) was higher than in other studies [26], while the proportion of inpatients admitted to the ICU (4.1\%) was lower than that reported in the literature [14]. In this situation, the use of the CURB-65 score could add support to clinical judgement regarding the need for hospital admission or intensive care management.

In conclusion, a simple severity assessment tool, the CURB-65 score, accurately classifies patients with CAP into different management groups: patients with CURB-65 scores of 0 who are at very low risk of mortality $(0 \%)$ and who, thus, may be suitable for home treatment; patients with scores of 1 who are at a relatively low risk of mortality $(1.1 \%)$ and who also may be suitable for home treatment, but who need additional criteria for admission decision; patients with scores of 2 who are at intermediate risk of mortality $(7.6 \%)$ and who should be considered for short-stay inpatient treatment; and patients with scores $>2$ who are at high risk of mortality $(26.7 \%)$ and who should be managed as having severe pneumonia. These four groups correlate significantly with key CAP management points: 1) admission decision criteria; 2) the timing of the switch from intravenous to oral antibiotics; and 3) discharge from the hospital. The CURB-65 score can also be used as a severity adjustment measure. Likewise, the CRB-65 score (which omits the blood urea result) may help general practitioners in the community decide when to hospitalise a patient with CAP.

Further research in the form of prospective studies is needed to determine if the CURB-65 (Confusion, Urea $>7 \mathrm{mmol} \cdot \mathrm{L}^{-1}$, Respiratory rate $\geqslant 30 \cdot \mathrm{min}^{-1}$, low Blood pressure, and age $\geqslant 65 \mathrm{yrs}$ ) score improves outcomes. In the meantime, the consistency of the findings of the current study with those of LIM et al. [9] suggest that this rule can be applied with some confidence in current practice while further prospective evaluation is underway.

\section{ACKNOWLEDGEMENTS}

The support of staff members of the different services is appreciated. The authors thank P.J. Skerrett for his assistance editing the manuscript. All statistical analysis was reviewed by 
I. Arostegui, Departamento de Matemática Aplicada, Estadística e Investigación Operativa, Universidad del País Vasco, Lejona, Spain.

\section{REFERENCES}

1 Neill AM, Martin IR, Weir R, et al. Community acquired pneumonia: aetiology and usefulness of severity criteria on admission. Thorax 1996; 51: 1010-1016.

2 Woodhead MA, Macfarlane JT, McCracken JS, et al. Prospective study of the aetiology and outcome of pneumonia in the community. Lancet 1987; 1: 671-674.

3 Almirall J, Bolíbar I, Vidal J, et al. Epidemiology of community-acquired pneumonia in adults: a populationbased study. Eur Respir J 2000; 15: 757-763.

4 Angus DC, Marrie TJ, Obrosky DS, et al. Severe community-acquired pneumonia: use of intensive care services and evaluation of American and British Thoracic Society diagnosis criteria. Am J Respir Crit Care Med 2002; 166: 717-723.

5 McQuillan P, Pilkington S, Allan A, et al. Confidential inquiry into quality of care before admission to intensive care. BMJ 1998; 316: 1853-1858.

6 Fine MJ, Auble TE, Yealy DM, et al. A prediction rule to identify low-risk patients with community-acquired pneumonia. N Engl J Med 1997; 336: 243-250.

7 España PP, Capelastegui A, Quintana JM, et al. A prediction rule to identify allocation of inpatient care in community-acquired pneumonia. Eur Respir J 2003; 21: 695-701.

8 Marras TK, Gutierrez C, Chan CK. Applying a prediction rule to identify low-risk patients with community-acquired pneumonia. Chest 2000; 118: 1339-1343.

9 Lim WS, van der Eerden MM, Laing R, et al. Defining community-acquired pneumonia severity on presentation to hospital: an international derivation and validation study. Thorax 2003; 58: 377-382.

10 British Thoracic Society. BTS guideline for management of community acquired pneumonia in adults. Thorax 2001; 56: Suppl. 4, 1-64.

11 Hanley JA, McNeil BJ. The meaning and use of the area under a receiver operating characteristic (ROC) curve. Radiology 1982; 143: 29-36.

12 Hanley JA, McNeil BJ. A method of comparing areas under receiver operating characteristic curves derived from the same cases. Radiology 1983; 148: 839-843.

13 Aujesky D, Auble TE, Yealy DM, et al. Prospective comparison of three validated prediction rules for prognosis in community-acquired pneumonia. Am J Med 2005; 118: 384-392.

14 Ewig S, Schäfer H, Torres A. Severity assessment in community-acquired pneumonia. Eur Respir J 2000; 16: 1193-1201.
15 Ewig S, Ruiz M, Mensa J, et al. Severe community-acquired pneumonia: assessment of severity criteria. Am J Respir Crit Care Med 1998; 158: 1102-1108.

16 Leroy O, Devos P, Guery B, et al. Simplified prediction rule for prognosis of patients with severe community-acquired pneumonia in ICUs. Chest 1999; 116: 157-165.

17 Kaplan V, Angus DC, Griffin MF, et al. Hospitalized community-acquired pneumonia in the elderly: ageand sex-related patterns of care and outcome in the United States. Am J Respir Crit Care Med 2002; 165: 766-772.

18 Díaz A, Alvarez M, Callejas C, Rosso R, Schnettler K, Saldías F. Clinical picture and prognostic factors for severe community-acquired pneumonia in adults admitted to the intensive care unit. Arch Bronconeumol 2005; 41: 20-26.

19 Ewig S, de Roux A, Bauer T, et al. Validation of predictive rules and indices of severity for community acquired pneumonia. Thorax 2004; 59: 421-427.

20 Niederman MS, Mandell LA, Anzueto A, et al. Guidelines for the management of adults with community-acquired pneumonia. Diagnosis, assessment of severity, antimicrobial therapy, and prevention. Am J Respir Crit Care Med 2001; 163: 1730-1754.

21 Halm EA, Fine MJ, Marrie TJ, et al. Time to clinical stability in patients hospitalized with community-acquired pneumonia: implications for practice guidelines. JAMA 1998; 279: 1452-1457.

22 Ramirez JA, Vargas S, Ritter GW, et al. Early switch from intravenous to oral antibiotics and early discharge: a prospective observational study of 200 consecutive patients with community-acquired pneumonia. Arch Intern Med 1999; 159: 2449-2454.

23 Rhew DC, Hackner D, Henderson L, et al. The clinical benefit of in-hospital observation in "low-risk" pneumonia patients after conversion from parenteral to oral antimicrobial therapy. Chest 1998; 113: 142-146.

24 Capelastegui A, España PP, Quintana JM, et al. Improvement of process-of-care and outcomes after implementing a guideline for management of communityacquired pneumonia: a controlled before-and-after study. Clin Infect Dis 2004; 39: 955-963.

25 Meehan TP, Weingarten SR, Holmboe ES, et al. A statewide initiative to improve the care of hospitalized pneumonia patients: The Connecticut pneumonia pathway project. Am J Med 2001; 111: 203-210.

26 Marrie TJ, Lau CY, Wheeler SI, et al. A controlled trial of a critical pathway for treatment of community-acquired pneumonia. JAMA 2000; 283: 749-755.

27 Chu LA, Bratzler DW, Lewis RJ, et al. Improving the quality of care for patients with pneumonia in a very small hospital. Arch Intern Med 2003; 163: 326-332. 